\title{
LA GENERATION Y EN EGYPTE SES BESOINS ET SES CAPACITES
}

\author{
ETUDE PRESENTEE PAR \\ HANAN WAGUIH GOUDA
}

MAITRE DE CONFERENCE A L'ECOLE DE MANAGEMENT ET DE

TECHNOLOGIE, FILIERE FRANCOPHONE.

ACAdemie Arabe des SCIENCES, de La TeChNOLOGIE et dU

TRANSPORT MARITIME.

DIRECTEUR DE LA COOPERATION INTERNATIONALE.

مجلة الدراسات التربوية و الانسانية ـ كلية التربية ـ جامعة دمنهور

المجلد الخامس العدد (4)- الجزء الثالث- لسنة 2013 


\section{Ses besoins et ses capacités}

\section{La génération Y en Egypte :}

Le terme génération Y désigne les personnes nées entre la fin des années $\underline{1976}$ et le milieu des années 1994 dont les parents sont souvent des baby-boomers ${ }^{1}$. Cette génération tire son nom de la génération précédente, nommée génération $\mathrm{X}$. D'autres termes équivalents existent, dont enfants du millénaire ou les diminutifs GénY et Yers. Les Américains utilisent également l'expression «digital natives » ou « net génération » pour pointer le fait que ces enfants ont grandi dans un monde où l'ordinateur personnel et l'Internet sont devenus de plus en plus accessibles.

Un concept occidental était à la base de cette nomination car cette catégorisation était essentiellement valable pour les pays occidentaux, bien que certaines caractéristiques soient vraies plus largement, du fait d'éléments géopolitiques majeurs. Elle s'est étendue un peu partout dans les sociétés développées qui renfermaient des traits plus au moins similaires dans l'environnement des jeunes et cela revient au fait que : Ils étaient suffisamment jeunes lors de l'introduction massive de l'informatique grand-public et de l'électronique portable (téléphonie mobile, photo numérique, GPS) pour en avoir acquis une maîtrise intuitive qui dépasse généralement celle de leurs parents (d'où le nom de « digital natives »).

Ils sont nés avec les débuts de l'intérêt du grand-public pour l'écologisme (qui était précédemment l'affaire d'une minorité, et souvent assimilée à l'extrême gauche).

Ils sont nés alors qu'IBM avait choisi le système d'exploitation de Microsoft pour son $\underline{\mathrm{PC}}$.

La mondialisation et la globalisation connues à ce siècle ont crée un effet de standardisation sur cette tranche du même âge : ils sont plus ou moins identiques et comportent les mêmes capacités et éprouvent les mêmes besoins.

De cela, est né une volonté de poser la problématique suivante :

\footnotetext{
${ }^{1}$ Les « Baby Boomers » sont les personnes nées entre 1946 et 1964, c'est une nomination américaine utilisée dans un contexte culturel désignant des personnes qui redéfinissent les valeurs traditionnelles de la génération précédente mais en restant fidèles aux mœurs et culture de leur ère.
}

635 
1. Avec le développement de la GEN Y, quels sont les nouveaux besoins des jeunes?

2. Quel rôle devraient avoir les institutions éducatives envers cette génération?

3. Comment la socialisation peut-elle influencer positivement cette génération?

Nous avons élaboré un questionnaire en anglais -ci-joint en annexespour couvrir un grand nombre de jeunes et pour ne pas limiter la recherche sur des francophones issues de classe sociale identique. Ce questionnaire a été distribué à un large nombre de jeunes de niveau social et éducatif différents. Les résultats de ce questionnaire nous ont aidés à développer notre conception pour essayer de prouver à la fin de cette recherche que :

1.les besoins des jeunes de la GEN Y sont proportionnels au développement de nouvelles technologies et à la globalisation.

2. la société Egyptienne devrait intégrer d`avantage la catégorie GEN Y pour qu ils puissent avoir une chance dans le marché du travail.

3. le système éducatif par obligation et responsabilité doit s'adapter aux exigences de la GEN Y.

\section{Qu'est-ce que la Génération Y?}

Plusieurs traits caractérisent la génération Y. D'abord, elle se situe dans une transition sociale, du déclin de l'impérialisme colonial à la chute du mur de Berlin (qui marqua la fin de la Guerre froide). Située juste après les baby-boomers (environ 1946-1959), cette génération a vécu un creux de vague au niveau professionnel, trouvant difficilement des emplois stables et bien rémunérés. Les formes nouvelles de précarité générationnelle lui sont spécifiques, en particulier dans les pays du sud de l'Europe. Il reste cependant important de dire que quelques caractéristiques dépendent plus largement du contexte politique et social même au sein d'un même pays.

Sous l'influence du numérique, d'internet, du téléphone mobile, la société a commencé à changer sous nos yeux sans qu'on en mesure spontanément la profondeur. Toute une génération se sert encore des outils numériques comme d'accessoires, cela est une vérité, mais la génération montante, elle, «s'en sert comme d'un élément central de 
communication, d'éducation, de distraction. Quand elle aura pris le pouvoir, les fonctionnements vont changer en profondeur $»^{2}$

\section{Culture générale de la génération $Y$}

Comme toute génération, son identité se construit autour des apports culturels reçus dès le plus jeune âge. Cette génération a largement grandi devant la télévision, et a vu l'arrivée en masse des séries d'animation japonaises. La vente de coffrets vidéo, ou d'autres en original ou traduits concernant les séries datant d'une vingtaine d'années témoigne de la nostalgie de cette génération pour la télévision qui l'a fortement influencée.

Cette catégorie privilégiée est naturellement plus à l'aise que les précédentes avec les technologies de l'information, et Internet en particulier. Elle peut être associée à l'ensemble des technologies et applications que l'on nomme aujourd'hui le Web 2.0. Chacun a accès à des outils de création et de communication dont les générations précédentes ne pouvaient que rêver. Ainsi, par exemple, écrire un livre dans les années 1970 nécessitait de le taper à l'aide d'une machine à écrire et à démarcher des éditeurs, ce qui rendait la diffusion des ouvrages plutôt incertaine. Aujourd'hui, on peut écrire sur son site web personnel (blog ou autre) depuis n'importe quel ordinateur, la diffusion du contenu étant immédiate.

La génération précédente a pu s'extasier devant les progrès constants réalisés par l'industrie audiovisuelle et ses effets spéciaux. Cependant, certaines études, ont démontré ${ }^{3}$ qu'une partie de la génération Y, les 1625 ans, consomment plus qu'ils ne développent les nouvelles technologies.

Pour la génération $Y$, qui est née après des films cultes tels que $\underline{\text { Star }}$ $\underline{\text { Wars, }}$, et était jeune pour d'autres plus récents comme The Matrix, les progrès vont de soi, et plus rien ne peut être graphiquement «étonnant », dans la mesure où « tout est possible », d'un dinosaure à la destruction d'une planète.

\footnotetext{
${ }^{2}$ Pat BATES, Mike BIERE, Rex WIEDERANDERS, Alan MEYER, Bill WONG, « $\underline{\text { Une }}$ nouvelle intelligence pour une planète plus intelligente », éd. MC Presse Online, France, 2009, p.97

${ }^{3}$ Ibid., p.102
} 
Les dates admises pour la génération $\mathrm{Y}$ correspondent à l'arrivée des jeux vidéo dans les foyers des familles aisées ; c'est donc la première génération à en avoir profité dès le plus jeune âge. Elle a donc grandi avec les effets positifs et négatifs liés à leur pratique. Il est important de signaler que tous ces effets sont source de débat, que ce soit au niveau de l'agressivité, des réflexes ou de la représentation dans l'espace, etc. «Quand on sait à quel point la rapidité de l'accès à l'information est grande avec le net, on se doute bien que la génération Y ne supporte pas d'attendre : ils veulent des résultats rapides, qu'il s'agisse de leur travail ou de celui des autres. Ils vont donc droit au but. ». Ils veulent communiquer directement avec les bons interlocuteurs et ne s'embarrassent pas des procédures intermédiaires qui leur font perdre leur temps

\section{L'éducation}

Les jeunes à ce stade de l'enseignement secondaire et universitaire, considéré comme leétape la plus importante de leur socialisation, se heurtent à la ruée vers la liberté qu'ils n'ont pas assez expérimentée: ils ont tout simplement envie de se sentir libre de tout contrôle parental. Ils comportent en eux une énergie explosive qui doit être bien canalisée. Donc, si l'éducation de ses jeunes n'était pas assurée par une famille qui n'assume pas la charge et les responsabilités matérielles et/ou sociales, les étudiants sont souvent plus disposés à payer le risque.

A travers la vie sur le campus- pour de nombreux étudiants duniversités dans les pays en développement- commencent les expériences de l'indépendance et de la vie réelle. Ces expériences seront accompagnées par des caractéristiques de l'impact physiologique et psychologique sur les attitudes sociales des étudiants d'où la nécessité de l'autonomie et des expériences personnelles et de la tendance à la révolte contre l'autorité.

Etape importante et critique portant deux tranches :

La communauté étudiante est caractérisée par une communication facile et par une transmission des idées sans pression en présence de toutes diversité idéologique: il faut donc bien investir ces atouts positivement.

Il est vrai que les familles les plus aisées sont celles dont les enfants le plus souvent s'inscrivent à l'université, ce qui est un point commun, à titre d'exemple, entre les Etats Unis et l'Egypte. Les frais de la plupart

\footnotetext{
${ }^{4}$ Jean- Pierre Helfer, Michel Kalika, Jacques Ossoni, « Management, stratégie et Organisation », éd. Vuibert, France, 2010, p.349.

doi: 10.12816/0004235 
des universités européenne quant à eux, sont extrêmement chers tandis qu'en Egypte l'université publique est presque gratuite. En effet, l'accès aux études supérieures augmente avec les revenus, comme on peut le constater dans l'analyse faite sur les Etats-Unis ci-dessous. ${ }^{5}$

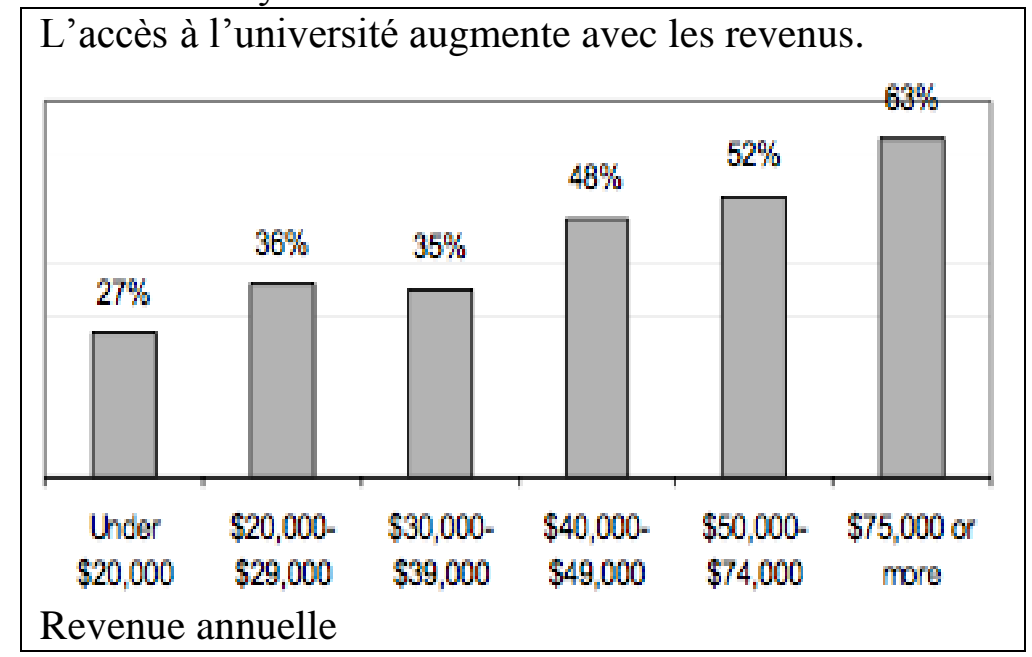

La majorité des familles avec des enfants entre 18 et 14 ans et des revenus d'environ $10000 \$$ ou plus ont au moins un enfant à l'université. Les critères de réussite à l'université ont mis en place une compétition pour les meilleures écoles et les meilleurs emplois. Un minimum de 4 ans d'études universitaires validés est requis pour la plupart des postes. L'idée générale veut que le diplôme ne garantisse pas une meilleure carrière mais permet d'y accéder par la grande porte. Les diplômes universitaires ayant pris tellement de poids dans la balance, de longues études sont devenues incontournables pour obtenir le poste désiré.

A ce stade d'étude, nous nous efforcerons de démontrer à quel point une contradiction flagrante se trouve au sein de cette génération en Egypte, génération étalée sur plusieurs niveaux de sociétés. Et c'est le cas de la situation en Egypte.

Tout d'abord voyons les propriétés de la scène de l'enseignement universitaire en Egypte à travers laquelle nous allons tenter d'expliquer les raisons pour lesquelles un fossé énorme s'est installé entre les jeunes d'une même génération, et qui de plus, se heurtent à une contradiction

\footnotetext{
${ }^{5}$ Elisabeth Bothom, "How generation Y works":The Millennial: Americans Born 1977 to 1994; Bureau of Census School, éd, IERPI, Québec, 1999, p.54.
}

\section{9}


flagrante quant aux apports disproportionnés offerts à cette même génération en Egypte. Preuve en est les conflits actuels et les différences d'opinions et de points de vue chez les jeunes à l'issue de la révolution du 25 Janvier 2011 et ce qui la suivis de problèmes politiques et sociaux. Nous montrerons comment les différents niveaux d'éducation en Egypte ont causé la naissance de 3catégories de génération dans une seule.

En Egypte, la génération [Y]se divise en...

\begin{tabular}{ccc}
\hline Y- & $|\mathbf{Y}|$ & $\mathbf{Y}+$ \\
\hline -Celle qui émane des & -Ceux des classes & -Ceux des classes \\
familles illettrées & défavorisées. Ils & favorisées. Ils \\
des milieux & suivent leur & suivent leur \\
ruraux ou des & cours dans un & cours dans un \\
bidonvilles. & effectif & environnement \\
& extrêmement & extrêmement \\
& élevé, avec peu & élevé avec un \\
& d'outils & effectif modeste. \\
& pédagogiques. & \\
\hline
\end{tabular}

[Y-] : celle qui émane des familles illettrées des milieux ruraux ou des bidonvilles; ils n'ont aucun accès à l'éducation sous toutes ses formes, ils sont privés du savoir. Leur environnement ne leur permet pas d'accéder aux universités et parfois pas aux écoles, et la plupart du temps les familles ne sont pas enthousiastes à cette idée. Ils seront donc au même niveau que leurs parents : les garçons apprennent le travail du père, les filles apprennent à cuisiner et à faire le ménage.

[Y] : ceux des classes défavorisées ; ils suivent leur cours universitaires dans un effectif extrêmement élevé; qui atteint des fois 1200 étudiants dans une seule salle, les supports éducatifs ne sont pas favorables à l'apprentissage, et la plupart du temps ne permettent pas aux jeunes de saisir l'information ni de l'appliquer dans un contexte extérieur à la salle de cours.

$[\mathrm{Y}+]$ : ceux des classes favorisées, ils suivent leur cours dans un environnent extrêmement adéquat, dans des écoles ou universités privées avec un effectif modeste, avec un accès à internet, aux langues étrangères et a des supports multimédias. Les jeunes des familles aisées qu'on appelle $\mathrm{Y}+$ possèdent les vraies caractéristiques de leur ère.

Si nous revenons en arrière, on remarque que l'école est à la base de ce fossé. Déjà dans l'Egypte ancienne, les parents inculquaient à leurs enfants plusieurs principes éducatifs, des attitudes morales et des approches de la vie dès leur plus jeune âge. Ils recevaient la base de leur éducation au sein de la famille. L'éducation égyptienne couvrait à la fois l'enseignement général délivré à un enfant et sa formation pour une vocation particulière. L'éducation des garçons était principalement 
confiée à leurs pères, les mères étaient responsables de l'éducation des filles. L'éducation dans l'Egypte ancienne variait selon les classes sociales. Les jeunes hommes ne choisissaient généralement pas leurs propres carrières. Au lieu de cela, ils suivaient généralement la profession ou le commerce familial, même pour les plus hautes fonctions du territoire. Les filles de familles moins nobles apprenaient à gérer un foyer, à chanter, à danser et à jouer des instruments de musique. Les enfants de fermiers et de pêcheurs avaient une éducation encore moins soutenue. Ils apprenaient à semer, à glaner et à récolter. Les artisans apprenaient à leurs enfants les bases de leur métier. Les enfants de la classe dirigeante étaient éduqués par des professeurs spécialisés. Les enfants de classe moyenne étaient éduqués dans les temples sous les auspices d'un professeur particulier, tout à l'image du système scolaire public qui est en besoin d'une reforme immédiate. Actuellement, le taux d'analphabétisme est très élevé : $45 \%$ de la population totale ne savent ni lire ni écrire, ceci est dû en première partie à la forte croissance démographique. Les classes des écoles sont passées donc d'un effectif de 60 élèves à un effectif de 80 élèves dans le public. D'autre part le manque de ressources se fait ressentir ; les locaux des écoles sont trop peu nombreux et les méthodes totalement archaïques.

C'est l'environnement dans lequel grandissent [Y-] et [Y] jusqu'à l'aboutissement à l'enseignement universitaire gouvernemental pour quelques-uns.

En Egypte nous pouvons parler de plusieurs différences concernant le système de l'enseignement dans les établissements universitaires. La méthode, les supports, le cursus même et l'atmosphère qui $\mathrm{y}$ règnent sont différents. Et toutes ces différences marquent la génération Y, sujet d'étude

La gravité de la situation réside dans le fait que la période scolaire et universitaire sont les étapes les plus importantes de la socialisation des jeunes, et se situent dans la vie à la fin de l'enfance et l'adolescence au début et avant la pleine maturité. «Cette période représente une source de renouveau et de changement et ne devrait donc pas se limiter à la notion de la jeunesse considérée comme une catégorie d'âge ou les caractéristiques sociales ou anatomiques imposent un mode d'emploi 
psychologique et comportemental $»^{6}$, mais plutôt une tranche importante de l'édifice dans le tissu social.

Face à cela, nous trouverons au sein de la même société les [Y+].

Ce sont les jeunes issus du système scolaire privé: langues, système international, professeurs étrangers et supports multimédias, vrai contexte de génération Y mondiale

Eux $[\mathrm{Y}+]$, ils ont un esprit plus libéral, plus abstrait que concret (raisonnement), plus mur (stabilité émotionnelle), plus respectueux (Conscience de la règle), plus audacieux et socialement aventureux (audace sociale), plus sensibles et sentimentaux (sensibilité), plus ouverts au changement et à l'expérimentation (ouverture au changement), et plus organisés et disciplinés (perfectionnisme) ceci revient également à leur vie familiale confortable "J'ai observé avec une attention particulière les collaborateurs qui étaient créateurs de la plus grande plus-value pour notre entreprise : il s'agissait des employés de la génération $\mathrm{Y}^{7}$

La cohésion familiale est présente et ancrée dans le quotidien de [Y+]. La Génération égyptienne a été élevée par des "parents actifs". Période définie par les termes de psychologie pédiatrique en tant que prédominante et comme une éducation parentale dispensée : il s'agit de la décennie de l'enfant. Ces parents ont passé plus de temps avec leurs enfants que ceux des générations précédentes. Tout comme les « vrais » de la Génération [Y] mondiale ceux qui voient l'enfant comme le centre de la famille. Les pères ont le facteur «enfant» dans l'équation et les compagnies réalisent que le temps passé en famille hors du travail est très important. Contrairement à la génération précédente - la Génération $\mathrm{X}$ - ceux dont les enfants n'étaient pas livrés à eux même pour prendre des décisions clefs; les parents de la Génération [Y+] sont très concernés. Ils s'impliquent dans la vie quotidienne et dans leurs décisions. «Leurs parents les aident à planifier leurs objectifs, prennent part à leurs activités, accordent l'intérêt et le soutien que leurs enfants méritent ${ }^{8}$

Plus de la moitié des familles de la génération $\mathrm{Y}$ en mondiale dînent ensemble 7jours par semaine. Ce qui permet aux enfants et parents de discuter autour d'une table de sujets importants. C'est le même cas en Egypte lorsqu'il s'agit de familles aisées.

\footnotetext{
${ }^{6}$ D.Palmer \& P. Mullaney, Building better alliance in «Out look journal $2 », 2011$, p. 53

${ }^{7}$ Karl M. Kapp, Tools and Techniques for Transferring Know How from Boomers to Gamers in" Global Business and Organizational Excellence", Volume 26, USA, OEC,2010, p.23

8 Anne kruger \& Baran Tuncer, "An Empirical test of the infant Industry Argument", USA, ed. American, Economic review, 1910, p. 129
} 
La majorité des enfants dinent avec leurs parents tous les jours.

Bureau of Census ${ }^{9}$

0 fois dans la semaine

1-2 fois par semaine

3-6 fois par semaine

7 fois par semaine

Le sentiment de sécurité apporté par la solide implication de leurs parents conforte les membres de la Génération Y dans leur certitude de pouvoir accomplir pratiquement tout, et s'ils n'y parvenaient pas, ils pourraient toujours renter à la maison et recevoir aide et support.

Très tôt, la société égyptienne va se heurter à un conflit de catégories de génération : les [Y+] face aux [Y-] et aux [Y]. En quête d'une qualité de vie que seuls les riches et les célèbres connaissent, voulant ce qu'il y a de mieux et pensant le mériter, la Génération [Y+] ambitieuse marquera le futur de notre pays.

\section{Besoins de la génération [Y]}

Il faut cependant noter que les étudiants représentent un segment et une partie importante de tout édifice dans le modèle social de la société. Le développement personnel et communicatif de l'étudiant constitue un outil de développement, ce sont des atouts importants dans ce qu'on appelle « opérations d'assistance au processus éducatif ». Ce sont des soins qu'on donne à l'apprenant qui l'orientent pour qu'il travaille suivant une méthode aidant à atteindre plusieurs objectifs, à l'intégration et à atteindre des objectifs sociétaux. Les objectifs, les systèmes et les moyens de préparer les jeunes jouent un rôle clé dans la politique de

\footnotetext{
${ }^{9}$ Etablit selon les statistiques du Bureau of Census aux Etats Unis en 2000. Nous considérons ses statistiques applicables sur les générations actuelles en Egypte, étant en décalage d'une dizaine d'année de celles des Etats Unis.
}

643 
l'éducation de n'importe quel Etat, ce rôle est lié à sa politique de développement économique et social. Cette préparation vise à réaliser l'intégration de l'étudiant à l'université et la croissance de la conscience qui l'aide à contribuer à bâtir la société à travers son développement personnel :

A cet âge, le jeune d'une vraie génération [Y] renferme un certain nombre de besoins, on cite entre autres :

L'appartenance à la société - la nécessité de l'estime de soi - le besoin de sentir l'importance - la nécessité de se sentir en sécurité et la stabilité - la nécessité de participer aux décisions et décider de son sort - le besoin d'interagir - la nécessité de dialogue et de débat - le besoin pour le mouvement et l'activité - le besoin d'exprimer- l'innovation - le besoin de créer une relation fructueuse sociale - et, enfin, la nécessité de s'adapter à la vie sociale.

Des études sociales ont montré que la jeunesse moderne politique et égyptienne de la classe défavorisée appelée dans cette recherche [Y] t [Y-] sont dans un état d'aliénation de la société éducative internationale ce qui est dans le temps actuel très grave et est à la base de tout problème politique et économique .

L'affiliation à une communauté est une voie importante que le jeune égyptien pouvait entreprendre et considérée comme relation réciproque renfermant le droit et le devoir, l'obligation et la responsabilité d'où l'importance de développer des jeunes bien intégrés capables d'atteindre les objectifs de la communauté.

La maximisation du rôle de l'université comme une des institutions les plus importantes de socialisation pour les jeunes est également une des solutions.

Il ne s'agit plus donc d'une formation universitaire seulement, il s'agit d'un outil pour la préparation de la main-d'œuvre formée et qualifiée et éduquée. La formation universitaire sera selon cette optique la science associée à la connaissance ainsi que son application sur les différents aspects de la vie en société et donc la source principale des facteurs de production et de progrès dans la société.

Par conséquent, l'enseignement supérieur sera sans aucun doute le défi de l'excellence du prochain siècle en Egypte. 


\section{Tableaux explicatifs des résultats des questionnaires}

Les différences significatives entre les moyens des degrés des langues et de l'arabe :

\begin{tabular}{|l|l|l|l|l|l|l|}
\hline \multirow{2}{*}{} & \multicolumn{2}{|l|}{$\begin{array}{l}\text { Langues } \\
(\mathrm{n}=151)\end{array}$} & \multicolumn{2}{l|}{ Arabe $(\mathrm{n}=208)$} & $\begin{array}{l}\text { Valeur } \\
(\mathrm{t})\end{array}$ & $\begin{array}{l}\text { Niveau de } \\
\text { signification }\end{array}$ \\
\cline { 2 - 6 } & $\mathrm{m}$ & $\mathrm{p}$ & $\mathrm{M}$ & $\mathrm{P}$ & & \\
\hline $\begin{array}{l}\text { La vie personnelle } \\
\text { et familiale }\end{array}$ & 13.24 & 1.76 & 11.53 & 1.63 & 9.243 & 0.001 \\
\hline $\begin{array}{l}\text { L'éducation } \\
\text { L'utilisation de la } \\
\text { technologie } \\
\text { moderne }\end{array}$ & 25.43 & 1.40 & 9.61 & 1.39 & 1.176 & $\begin{array}{l}\text { Pas } \\
\text { mentionnée }\end{array}$ \\
\hline $\begin{array}{l}\text { Le travail et les } \\
\text { valeurs } \\
\text { professionnels }\end{array}$ & 18.09 & 1.55 & 16.19 & 2.11 & 9.135 & 0.001 \\
\hline
\end{tabular}

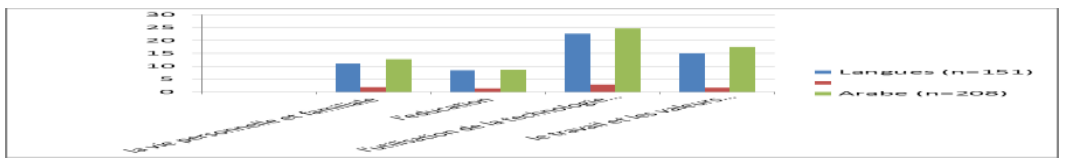

Les coefficients de corrélation entre les dimensions de la matrice de résolution de l'échantillon (langues) : $(\mathrm{N}=151)$

\begin{tabular}{|l|l|l|l|l|}
\hline & $\begin{array}{l}\text { La vie } \\
\text { personnell } \\
\text { e et } \\
\text { familiale }\end{array}$ & $\begin{array}{l}\text { L'éducati } \\
\text { on }\end{array}$ & $\begin{array}{l}\text { L'utilisation de } \\
\text { la technologie } \\
\text { moderne }\end{array}$ & $\begin{array}{l}\text { Le travail et } \\
\text { les valeurs } \\
\text { professionnels }\end{array}$ \\
\hline $\begin{array}{l}\text { La vie } \\
\text { personnel } \\
\text { le et } \\
\text { familiale }\end{array}$ & -1.76 & 11.53 & 1.63 \\
\hline $\begin{array}{l}\text { L'éducation } \\
0.309^{* *} \\
0.01\end{array}$ & 1.40 & 9.61 & 1.39 \\
\hline $\begin{array}{l}\text { L'utilisation } \\
\text { de la } \\
\text { technologie } \\
\text { moderne }\end{array}$ & $\begin{array}{c}0.125 \\
\text { Pas } \\
\text { mentionnée }\end{array}$ & 2.72 & 22.61 & 1.95 \\
\hline $\begin{array}{l}\text { Le travail et } \\
\text { les valeurs } \\
\text { professionne } \\
\text { ls }\end{array}$ & $\begin{array}{c}0.183^{*} \\
0.05\end{array}$ & 1.55 & 16.19 & 2.11 \\
\hline
\end{tabular}


Ressort clairement du tableau ci-dessus qu'il existe une corrélation statistiquement significative entre la vie personnelle et familiale et toutes les valeurs de l'éducation, de travail et les valeurs professionnelles, mais il n'y a pas de relation entre la vie personnelle et familiale et l'utilisation de la technologie moderne et on retrouve également une corrélation statistiquement significative entre l'éducation et l'utilisation de la technologie moderne et les valeurs professionnelles comme il existe une relation entre l'utilisation de la technologie moderne, le travail et les valeurs professionnelles.

Les coefficients de corrélation entre les dimensions de la matrice de résolution de l'échantillon (l'arabe) : (N=208)

\begin{tabular}{|c|c|c|c|c|}
\hline & $\begin{array}{l}\text { La vie } \\
\text { personnell } \\
\text { e et } \\
\text { familiale }\end{array}$ & L'éducation & $\begin{array}{l}\text { L'utilisation de la } \\
\text { technologie } \\
\text { moderne }\end{array}$ & $\begin{array}{l}\text { Le travail et } \\
\text { les valeurs } \\
\text { professionnels }\end{array}$ \\
\hline \multicolumn{5}{|l|}{$\begin{array}{l}\text { La vie } \\
\text { personnelle et } \\
\text { familiale }\end{array}$} \\
\hline L'éducation & $\begin{array}{l}0.006 \text { Pas } \\
\text { mentionné } \\
\text { e }\end{array}$ & L & & \\
\hline $\begin{array}{l}\text { L'utilisation de } \\
\text { la technologie } \\
\text { moderne }\end{array}$ & $\begin{array}{l}0.106 \\
\text { Pas } \\
\text { mentionné } \\
\text { e }\end{array}$ & $\begin{array}{l}0.221 * * \\
0.01\end{array}$ & 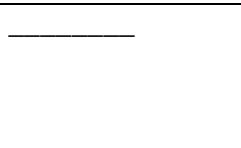 & \\
\hline $\begin{array}{l}\text { Le travail et les } \\
\text { valeurs } \\
\text { professionnelles }\end{array}$ & $\begin{array}{l}0.149 * \\
0.05\end{array}$ & $\begin{array}{l}0.082 \\
\text { Pas mentionnée }\end{array}$ & $\begin{array}{l}0.176^{*} \\
0.05\end{array}$ & - \\
\hline
\end{tabular}

Les différences significatives entre les moyens des degrés des langues et de l'arabe :

\begin{tabular}{|l|l|l|l|l|l|l|}
\hline & \multicolumn{2}{|l|}{$\begin{array}{l}\text { Langues } \\
\mathrm{(n}=151)\end{array}$} & \multicolumn{2}{l|}{ Arabe (n=208) } & Valeur (t) & $\begin{array}{l}\text { Niveau de } \\
\text { signification }\end{array}$ \\
\cline { 2 - 6 } & $\mathrm{M}$ & $\mathrm{p}$ & $\mathrm{m}$ & $\mathrm{p}$ & & \\
\hline $\begin{array}{l}\text { La vie } \\
\text { personnelle et } \\
\text { familiale }\end{array}$ & 11.00 & 1.86 & 12.69 & 1.74 & 8.724 & 0.001 \\
\hline $\begin{array}{l}\text { L'éducation } \\
\text { L'utilisation de }\end{array}$ & 8.46 & 1.40 & 8.62 & 1.39 & 1.068 & $\begin{array}{l}\text { Pas } \\
\text { mentionné }\end{array}$ \\
\hline $\begin{array}{l}\text { la technologie } \\
\text { moderne }\end{array}$ & 22.69 & 2.89 & 24.69 & 2.05 & 8.415 & 0.001 \\
\hline $\begin{array}{l}\text { Le travail et les } \\
\text { valeurs } \\
\text { professionnels }\end{array}$ & 15.06 & 1.65 & 17.49 & 2.51 & 11.041 & 0.001 \\
\hline
\end{tabular}




\section{Ouvrages :}

\section{Bibliographie}

1.Autissier, David, Faouzi Bensebaa, et Fabienne Boudier. L'atlas $d u$ management: L'encyclopédie du management en 100 dossier-clés. Édition : édition 2010-2011. Eyrolles, 2011.

2.Berberova, Nina Nikolaevna. L'accompagnatrice: roman. Paris: Actes Sud, 1985.

3.Bernhard, Thomas, Simon, Boris, et Josée Turk-Meyer. Gel. Paris: Gallimard, 1967

4. Dewey, John, Zask, Joëlle. Le public et ses problèmes. Paris: Gallimard, 2010.

5.George Orwell, Bernard Hoepffner. Ecrits politiques, 1928-1949 - broché Livre.

6.Joliot, Pierre. La recherche passionnément. Paris: Odile Jacob, 2001.

7.Kafka, Franz, Marthe Robert, Jean-Pierre Danès, et Claude David. Oeuvres complètes vol. 3 vol. 3. Paris: Gallimard, 1984.

8.Lévy, Maurice. Les 100 mots de la communication. Paris: Presses universitaires de France, 2006.

9.Mintzberg, Henry. Le management: voyage au centre des organisations. Paris: Éditions d'Organisation, 2004.

10.Nayar, Vineet. Employees First, Customers Second: Turning Conventional Management Upside down. Boston, Mass.: Harvard Business Press, 2010.

11.Rollot, Olivier. La génération Y. Paris: Presses universitaires de France, 2012.

12.Saba, Tania, Shimon L. Dolan, et Susan E Jackson. La gestion des ressources humaines tendances, enjeux et pratiques actuelles. Paris; [Saint-Laurent (Québec)]: Pearson éducation; Éd. du renouveau pédagogique, 2008.

13.Voltaire, et Alain Pons. Dictionnaire philosophique. Paris: Gallimard, 1994.

Actes de colloques :

1.Colloque Sillage de Kafka, Zard, Philippe, Université de Paris X: Nanterre, et Centre de recherche en littérature et poétique comparées, éd. Sillage de Kafka: actes du colloque « Sillage de Kafka » organisé à l'Université de Paris-X-Nanterre, 11 -13 mars 2004, dans le cadre $d u$ Centre de recherche en littérature et poétique comparées. Paris: Manuscrit, 2007.

\section{Sites web et Blog :}

3.Base de données de l'UNESCO, rubrique: éducation pour tous ; Quatrième Réunion du Groupe de haut niveau sur l'éducation pour Tous, Brésil 8-10 novembre 2004 
4. http://www.unesco.org/new/fr/education/themes/leading-theinternational-agenda/education-for-all/coordination-mechanisms/highlevel-group/4th-meeting-brasilia-brazil-8-10-november-2004/

5.Michel Serres, "Ce n'est pas une crise c'est un changement de monde » in le Monde, interview, 2012

6.http://www.lejdd.fr/Economie/Actualite/Serres-Ce-n-est-pas-une-crise-cest-un-changement-de-monde-583645

7.Social and Economic Characteristics of Students in American AcademicVolume

3, NCES 2002http://dbweb01.aft.org/pdfs/highered/academic/january07/Engle.pdf

8. Thibaut Le Pellec, "La génération Y dans le monde de l'entreprise" in Blog Knowtex, 2010

9.http://www.knowtex.com/nav/la-generation-y-dans-le-monde-de-lentreprise_5352

\section{Références}

1.Anne kruger \& Baran Tuncer, "An Empirical test of the infant Industry Argument", USA, ed. American, Economic review, 1910, p. 129

2.D.Palmer \& P. Mullaney, Building better alliance in "Out look journal $2 », 2011$, p. 53

3.Elisabeth Bothom, "How generation Y works": The Millennial: Americans Born 1977 to 1994; Bureau of Census School, éd, IERPI, Québec, 1999, p.54

4.Jean- Pierre Helfer, Michel Kalika, Jacque Ossoni, "Management, stratégie et Organisation », éd. Vuibert, France, 2010, p.349.

5.Karl M. Kapp, Tools and Techniques for Transferring Know How from Boomers to Gamers in" Global Business and Organizational Excellence", Volume 26, USA, OEC,2010, p.23

6.Pat BATES, Mike BIERE, Rex WIEDERANDERS, Alan MEYER, Bill WONG, "Une nouvelle intelligence pour une planète plus intelligente », éd. MC Press Online, France, 2009, p.97 


\section{Matériel et méthodes}

Cette étude a lieu à Alexandrie entre mars 2010 et mai 2010 . le public cible étant les jeunes nés entre 1976 et 1994 de deux sexes .

Un questionnaire a été lancé sur Internet (facebook) et aussi distribué par main propre.

L’échantillon était désigné tel que les catégories y+ et y- sont présentées selon leur état socioéconomique et le genre d’éducation scolaire (privéepublique) négligeant la catégorie $\{y\}$ la plupart n`étant pas scolarisée.

L'échantillon final consiste de :

$\mathrm{Y}+151$

$\mathrm{Y}-208$

Total $=359$

Le questionnaire comporte 4 sections :

1-La vie familiale

2-L’éducation scolaire

3-L`usage de la technologie

4-Le travail et les valeurs professionnels

Les résultats ont été statistiquement analysés.

Une copie du questionnaire est ajoutée en annexe.

Annexes:

Exemplaires en 7 pages en anglais. 
